\title{
Nonlinear absorption and refraction in near-detuned rubidium vapor
}

\author{
C. F. McCormick, D. R. Solli, R. Y. Chiao' ${ }^{1}$ and J. M. Hickmann ${ }^{2}$ \\ ${ }^{1}$ Department of Physics, University of California, Berkeley, California 94720-7300 \\ ${ }^{2}$ Departamento de Física, Universidade Federal de Alagoas, \\ Cidade Universitária, 57072-970, Maceió, AL, Brazil
}

\begin{abstract}
Using the z-scan technique, we have measured the self-induced absorptive and refractive nonlinear behavior of hot atomic rubidium vapor within the Doppler profile of the D2 line. We observe large nonlinear amplitude and phase effects with only tens of microwatts of incident power. Our results are in good agreement with numerical calculations based on an analytic model of a Doppler- broadened two-level system.
\end{abstract}


Atomic vapors are interesting nonlinear optical materials because their nonlinear coefficients depend strongly on detuning, and nonlinear effects can be observed with very low cw laser power. Nonlinear optics experiments in atomic vapors have included self-focusing, self-defocusing, selftrapping and self- bending [1, 2, 3, 4]; third-harmonic generation [5]; and four-wave mixing [6]. Several measurements of Kerr nonlinear coefficients have been reported in atomic vapors, for large detunings [7], and some recent attention has focused on employing quantum coherence to control atomic nonlinear properties [8].

There has also been recent discussion concerning the role of the nonlinearity of atomic rubidium vapor in light-by-light guiding [9]. Theoretical work using three-level [10] and five-level [11] models has been reported, both of which incorporate Doppler broadening numerically. The latter of these papers argues that the five-level model is necessary to accurately predict the self-action of a pump beam propagating in $\mathrm{Rb}$ vapor, and presents theoretical results on the index of refraction variation due to self-action.

In this work, we report a measurement of self-induced nonlinear absorption and refraction in hot atomic $\mathrm{Rb}$ vapor, within the Doppler profile of the D2 line $(\lambda=780 \mathrm{~nm})$. The nonlinearity is due to saturated atomic absorption, and only tens of microwatts are needed to get into the saturation regime for detunings within the Doppler profile. Inhomogenous Doppler broadening complicates the form of the nonlinearity and a Kerr model is insufficient to describe it. A fully analytic solution exists for the nonlinear behavior of a Doppler-broadened, two-level system, and it is reasonably tractable in the limit of small power-broadened linewidth compared with the Doppler width [12]. Using this model we successfully predict the beam propagation and self-action effects in our experiment. While a slight variant of this model has frequently been discussed in relation to nonlinear absorption in inhomogeneously broadened two-level systems, its application to selfaction refractive effects has been much rarer [13].

Our experimental setup is a standard z-scan configuration [14] (see Fig. 11). The primary beam is provided by a $10 \mathrm{~mW}$ tunable $\mathrm{cw}$ diode laser with a bandwidth $\delta \nu \approx 300 \mathrm{kHz}$. We measure its detuning to within $10 \mathrm{MHz}$ by interfering it on a fast photodiode with a second diode laser locked using saturation absorption spectroscopy to the crossover resonance $5 S_{1 / 2} F=3 \rightarrow 5 P_{3 / 2} F^{\prime}=$ 3, 4 of ${ }^{85} \mathrm{Rb}$ (in the D2 line), and computing a real-time fast Fourier transform of the photodiode signal. The multimode transverse profile of the experimental beam is regularized to a cylindrically symmetric, nearly-Gaussian shape by coupling it through a single-mode fiber with about $30 \%$ efficiency. The beam is focused by a lens to a Rayleigh range $z_{R} \approx 8 \mathrm{~mm}$ and is detected in the 


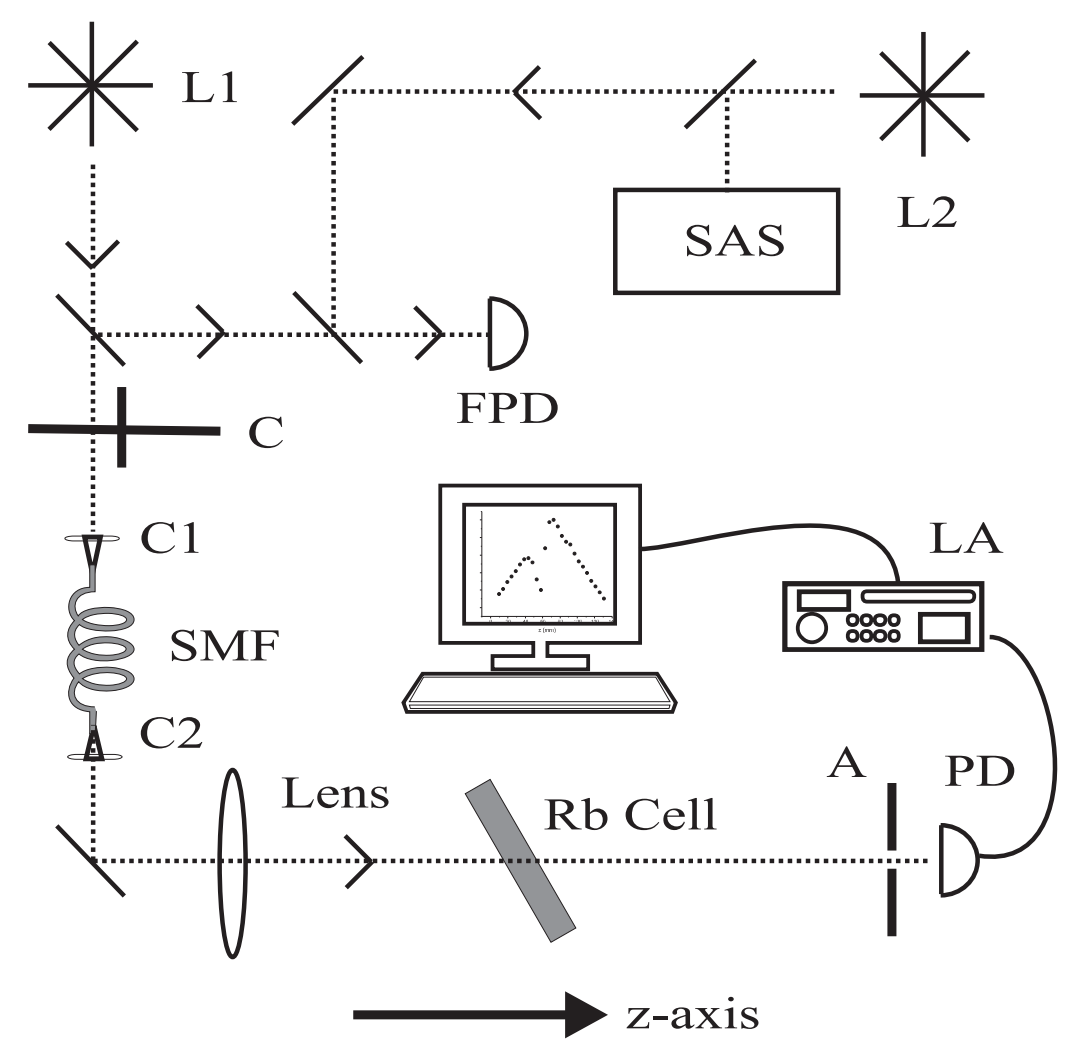

FIG. 1: Experimental configuration for the z-scan measurement. L1 and L2 = lasers 1 and 2; SAS = saturated absorption spectroscopy lock; $\mathrm{C}=$ chopper wheel; $\mathrm{C} 1$ and $\mathrm{C} 2=$ fiber coupling lenses; $\mathrm{SMF}=$ single mode fiber; $\mathrm{A}=$ aperture; $\mathrm{PD}=$ photodiode; $\mathrm{LA}=$ lock-in amplifier; $\mathrm{FPD}=$ fast photodiode.

far-field using a photodiode. An aperture is placed in front of the photodiode, centered on the experimental beam. The intensity noise of the beam at the detector is less than $0.5 \%$.

We place a thin $\left(\mathrm{L}=1 \mathrm{~mm} \ll z_{R}\right)$ optical vapor cell filled with natural-abundance $\mathrm{Rb}$ in the path of of the beam after the lens. It has no magnetic shielding and experiences only the geomagnetic field. The cell is heated to $78^{\circ} \mathrm{C}$ and tilted at $30^{\circ}$ to prevent etalon effects. It is mounted on a 250 $\mathrm{mm}$ translation stage moved along the length of the experimental beam by a computer-controlled stepper motor. To measure the noise in our system, we detune far off-resonance and record the transmission through the vapor cell as it is scanned $250 \mathrm{~mm}$ along the focused beam. For both fully open and $20 \%$ fluence apertures these scans show less than $\pm 1 \%$ transmission variation.

Using horizontal linear polarization and $45 \mu \mathrm{W}$ of power, we performed scans with the aperture both fully open and closed to $20 \%$ linear fluence, at detunings of $\pm 300 \mathrm{MHz}$ from the ${ }^{85} \mathrm{Rb}$ $F=3 \rightarrow F^{\prime}$ transition (see Figs. 2 3, 34). This detuning was chosen as representative because it is of the same order as the Doppler width of the transition, $\approx 380 \mathrm{MHz}$. With the aperture open, 


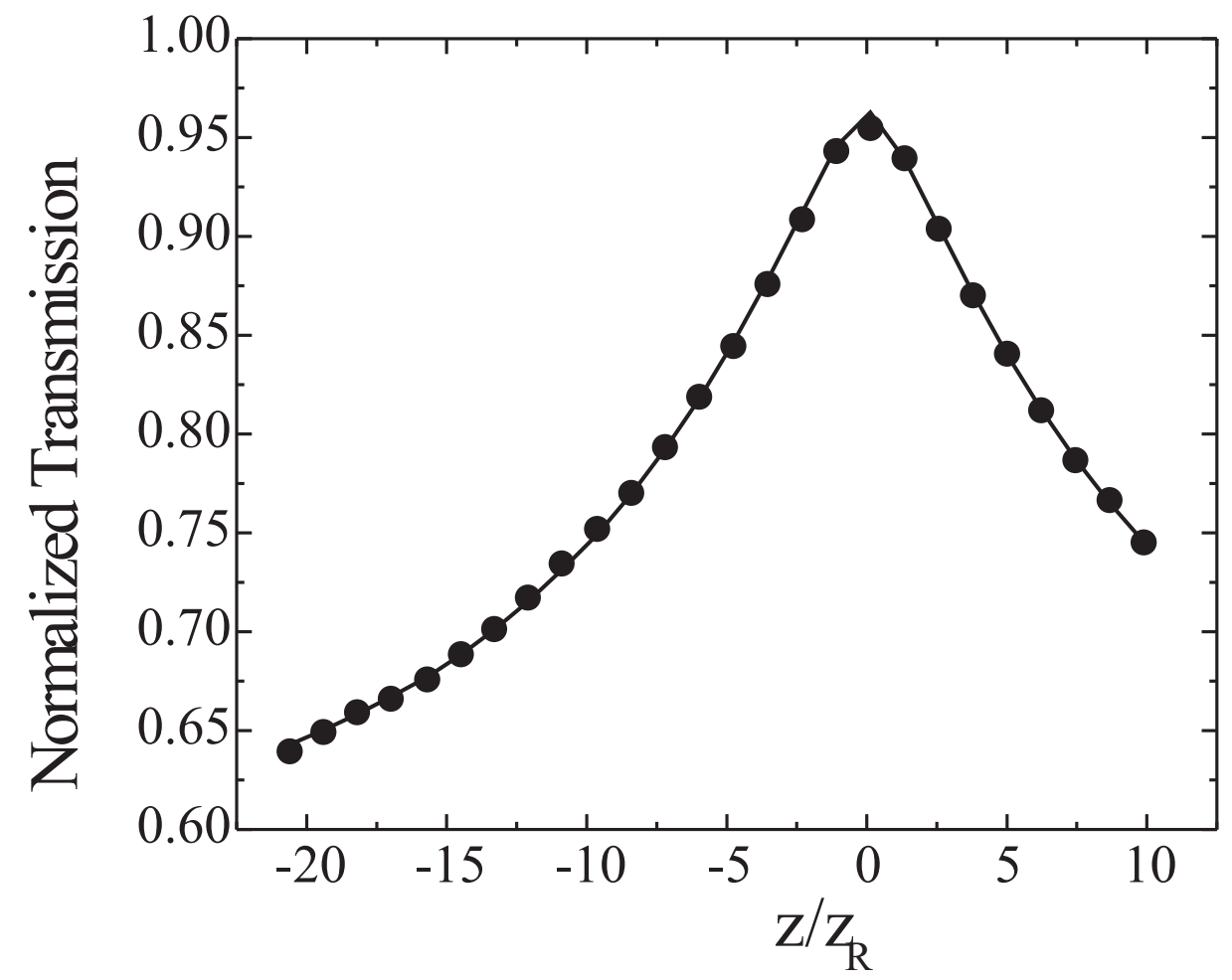

FIG. 2: Open-aperture scan at $300 \mathrm{MHz}$ to the blue of the ${ }^{85} \mathrm{Rb} \mathrm{F}=3 \rightarrow$ F' line, with fit from the Dopplerbroadened two-level model. The scan at $300 \mathrm{MHz}$ to the red is very similar.

there should be no refractive effects, and these scans display the qualitative behavior expected from a negative absorptive nonlinearity (i.e. absorption saturation). With the aperture closed, the nonlinear phase imprinted by the atoms on the beam will change the far-field beam width and be detected as an asymmetric transmission profile with $\mathrm{z}$, as we observe. The symmetry of these scans is that of a self-focusing (-defocusing) nonlinearity for blue (red) detuning, as expected.

To quantitatively fit the scans, we model the $\mathrm{Rb}$ vapor as an inhomogenously (Doppler) broadened two-level system [12]. The general expressions for the absorptive and refractive coefficients involve complex error functions, but can be considerably simplified when the power-broadening of the homogenous linewidth is small compared to the Doppler width. In this limit the absorption coefficient and index of refraction are given by

$$
\begin{gathered}
\alpha(x, I)=\alpha_{0}\left(\frac{e^{-x^{2}}}{\sqrt{1+I / I_{s}}}-\frac{2 a}{\sqrt{\pi}}(1-2 x F(x))\right) \\
n(x, I)=1-\alpha_{0} \frac{a c}{\omega}\left(x e^{-x^{2}} \sqrt{1+I / I_{s}}-\frac{1}{a \sqrt{\pi}} F(x)\right)
\end{gathered}
$$




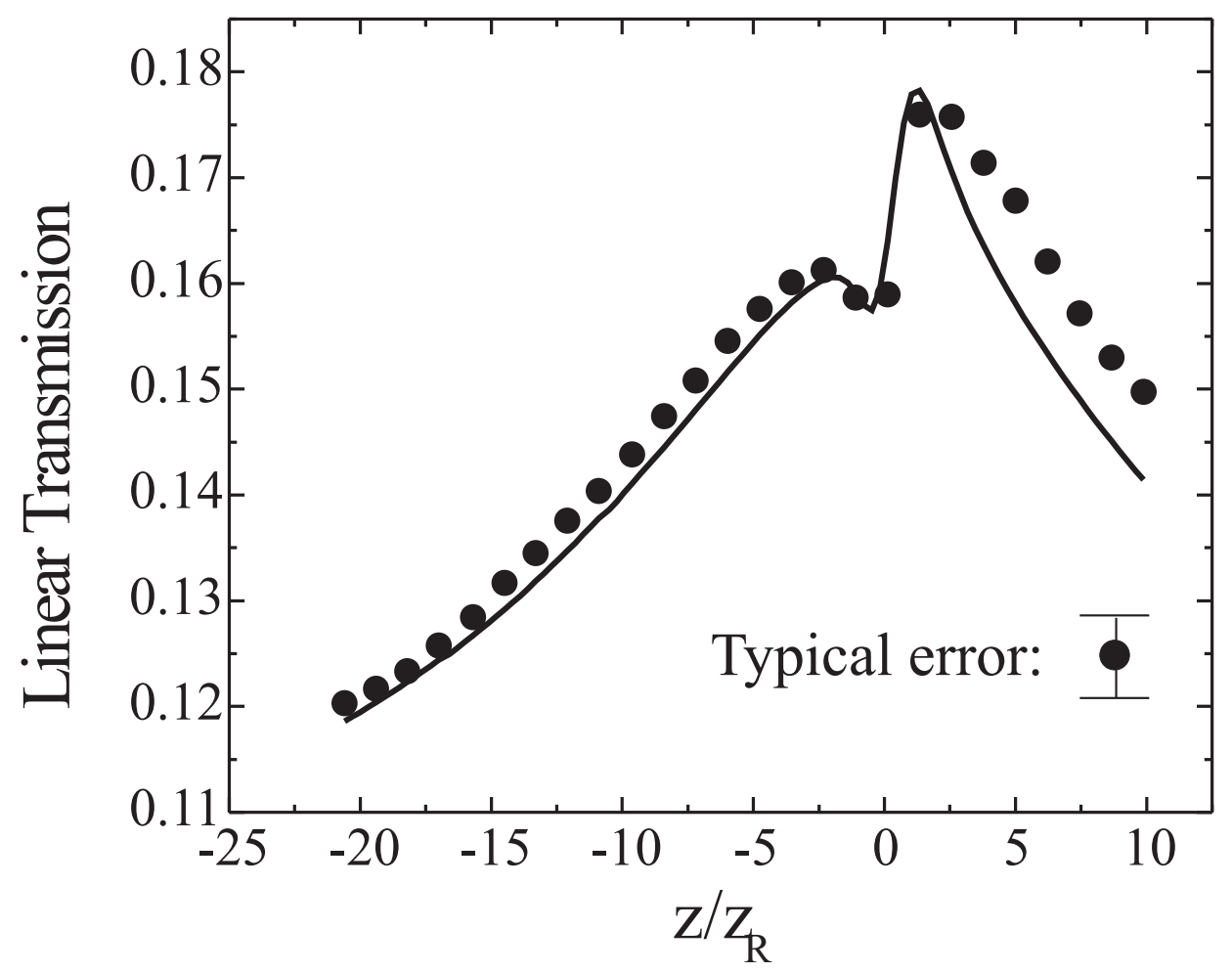

FIG. 3: Closed-aperture scan at $300 \mathrm{MHz}$ to the blue of the ${ }^{85} \mathrm{Rb} \mathrm{F}=3 \rightarrow \mathrm{F}$ ' line. The theory curve is calculated directly from the fit parameters found in the corresponding open scan, with no adjustable parameters. The typical systematic error mostly results from residual beam astigmatism and uncertainties about the aperture size.

Here $a$ is the homogeneous linewidth and $x$ is the detuning (positive for the red side), both normalized to the Doppler width. $I$ is the beam intensity, $I_{s}$ is the atomic saturation intensity, $\omega$ is the laser angular frequency and $\alpha_{0}$ is the linear absorption coefficient in the absence of Doppler broadening. The function $F(x)$ is Dawson's integral, given by [15]

$$
F(x)=e^{-x^{2}} \int_{0}^{x} e^{+t^{2}} d t .
$$

In the limit of low intensity, Eqs. 1 1 and 2 become a Kerr (third-order) nonlinearity.

In a true Doppler-broadened two-level system, $\alpha_{0}$ and $I_{s}$ depend only on the homogeneous linewidth, resonance frequency, atomic density and dipole moment. Because of the hyperfine structure and the two isotopes ${ }^{85} \mathrm{Rb}$ and ${ }^{87} \mathrm{Rb}$, our system is not truly two-level, so we allow $\alpha_{0}$ and $I_{s}$ to be parameters in our fit. Our fitting procedure performs a numerical integration of the beam through the cell at each radial point, using Eqs. 1 1)and2. The output beam is then propagated 


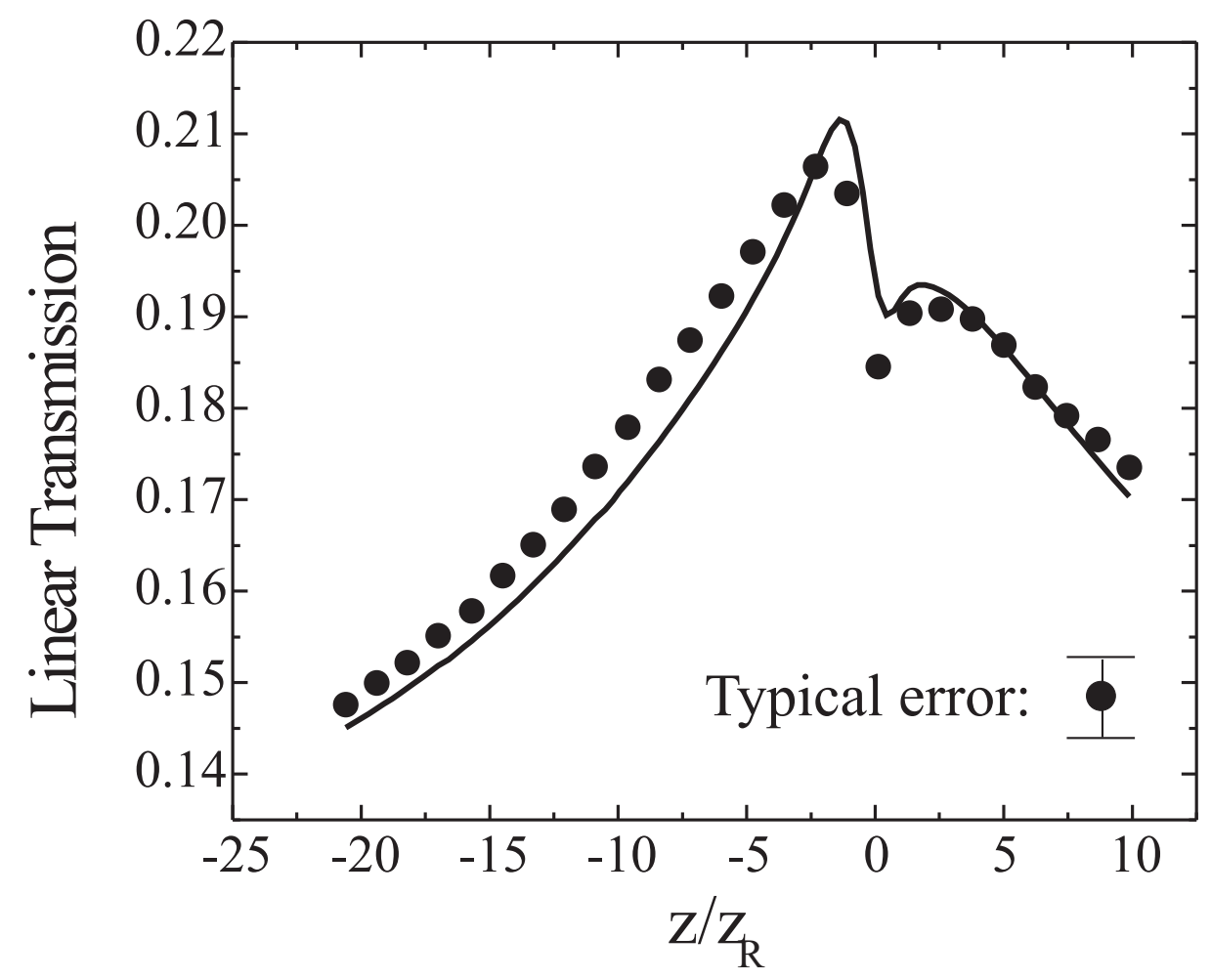

FIG. 4: As above, for a detuning of $300 \mathrm{MHz}$ to the red.

to the far-field detector using a quasi-fast Hankel transform [16]. We use enough radial points so that, with absorption and refraction turned off, our model gives unity transmission to better than $0.05 \%$. To estimate the numerical error in our integration, we perform the fits twice, doubling the number of integration steps in the second instance. The two fitting results differ by less than $2 \%$. It is worth noting that although our cell is "thin" in the sense that it is much shorter than the beam Rayleigh range, it is not thin compared to the atomic absorption length. This requires us to perform a full integration through the vapor, rather than simply imposing a single absorption and phase profile on the beam as it passes through the cell.

Our numerical fits give excellent agreement with the open-scan data, with parameters $I_{s}=$ $2.1 \mathrm{~mW} / \mathrm{cm}^{2}$ for both red and blue detuning, and $\alpha_{0}=9.4 \mathrm{~cm}^{-1}, 9.9 \mathrm{~cm}^{-1}$ for red and blue detuning, respectively (see Fig. 2). To model the closed-aperture scans, we use these parameters to calculate the transmission vs. $\mathrm{z}$ curves that should result for a $20 \%$ fluence aperture, finding good agreement within the systematic error (see Figs. 3, 4). The error is mostly from residual beam astigmatism and uncertainties about the aperture size. We emphasize that we do not fit or adjust any parameters to produce the theory curves for the closed-aperture scans. The agreement of the theory with the closed-aperture data is evidence of the Kramers-Kronig relations connecting 
the absorptive and refractive properties of this system, even though it is nonlinear [13]. For low intensities, the fit parameters imply a Kerr coefficient $\left(n_{2}\right)$ of $-5.5 \times 10^{-6} \mathrm{~cm}^{2} / \mathrm{W}$ for red detuning and $+5.8 \times 10^{-6} \mathrm{~cm}^{2} / \mathrm{W}$ for blue detuning. To check that we are in the limit in which Eqs. 1 and 2] are valid, we note that the ratio of the power-broadened homogeneous linewidth (38 MHz times the saturation parameter $\sqrt{1+I / I_{s}}$ ) to the Doppler width $(k v=2 \pi \times 380 \mathrm{MHz}, v$ is the most probable atomic velocity) is 0.4 for the axial center of the beam at $\mathrm{z}=0$, falling off quadratically in $\mathrm{z}$ and exponentially in the beam radius. Thus for calculations of the integrated transmission, Eqs. 1 and 2] are good approximations.

To determine the effect of the unshielded geomagnetic field we repeated the experiment with vertical linear polarization, finding scans that differ by about $10 \%$. Our fits produced values of $\alpha_{0}$ that were the same as for horizontal polarization to within $5 \%$, but the $I_{s}$ fits were consistently a factor of 2 larger. We attribute this to optical pumping effects, which can change the saturation intensity seen by the beam by changing the population of atoms in dark states. With these altered parameters, the fit of the model to the data is excellent, indicating that a detailed inclusion of optical pumping in the model is not needed.

The success of this analytic, Doppler-broadened, two-level model in describing self-induced absorptive and refractive effects suggests that more complicated models are not needed to understand self-action in atomic vapor. However, it is clear that this simple model fails to predict other important nonlinear effects such as cross-saturation and cross-phase modulation. Their correct treatment requires more complicated models that include the effects of more atomic levels.

In conclusion, we have used both open and closed z-scans with powers of only tens of microwatts to measure the absorptive and refractive nonlinearities of $\mathrm{Rb}$ vapor within the Doppler profile of the D2 line. Our open-aperture data are in excellent quantitative agreement with an analytic, Doppler-broadened two-level model. Parameters from the open-aperture fits produce theory curves for the closed-aperture data that fit well to within the systematic error. Our results imply that an analytic two-level model of the nonlinearity of $\mathrm{Rb}$ vapor is sufficient for describing selfaction effects, as long as the powers involved do not broaden the homogeneous linewidth to larger than the Doppler width.

This work was supported in part by ONR and NSF Grant No. 0101501. JMH thanks the support from Instituto do Milênio de Informação Quântica, CAPES, CNPq, FAPEAL, PRONEX-NEON, 
ANP-CTPETRO.

[1] D. Grischkowsky, "Self-focusing of light by potassium vapor," Phys. Rev. Lett. 24, 866-869 (1970).

[2] D. Grischkowsky and J. A. Armstrong, "Self- defocusing of light by adiabatic following in rubidium vapor," Phys. Rev. A 6, 1566-1570 (1972).

[3] J. E. Bjorkholm and A. Ashkin, "cw self-focusing and self-trapping of light in sodium vapor," Phys. Rev. Lett. 32, 129-132 (1974).

[4] G. A. Swartzlander, H. Yin, and A. E. Kaplan, "Self- bending of a cw laser beam in sodium vapor," Opt. Lett. 13, 1011-1013 (1988).

[5] J. F. Young, G. C. Bjorklund, A. H. Kung, R. B. Miles, and S. E. Harris, “Third-harmonic generation in phase-matched Rb vapor,” Phys. Rev. Lett. 27, 1551-1553 (1971).

[6] D. J. Harter, P. Narum, M. G. Raymer, and R. W. Boyd, "Four-wave parametric amplification of Rabi sidebands in sodium,” Phys. Rev. Lett. 46, 1192-1195 (1981); Y. Shevy and M. Rosenbluh, "Parametric four-wave mixing processes in sodium vapor," Opt. Lett. 12, 257-259 (1987).

[7] R. H. Lehmberg, J. Reintjes and R. C. Eckardt, "Negative nonlinear susceptibility of cesium vapor around $1.06 \mu \mathrm{m}$," Phys. Rev. A 13, $1095-1103$ (1976). S. Sinha, G. K. Bhowmick, S. Kundu, S. Sasikumar, S. K. S. Nair, T. B. Pal, A. K. Ray and K. Dasgupta, "A Z-scan study of nonlinear refraction in sodium vapor," Opt. Comm. 203, 427-434 (2002).

[8] Hai Wang, David Goorskey, and Min Xiao, "Dependence of enhanced Kerr nonlinearity on coupling power in a three-level atomic system," Opt. Lett. 27, 258-260 (2002).

[9] A. G. Truscott, M. E. J. Friese, N. R. Heckenberg, and H. Rubinsztein-Dunlop, "Optically written waveguide in an atomic vapor," Phys. Rev. Lett. 82, 1438-1441 (1999).

[10] Rakesh Kapoor and G. S. Agarwal, "Theory of electromagnetically induced waveguides," Phys. Rev. A 61, 053818 (2000).

[11] J. A. Andersen, M. E. J. Friese, A. G. Truscott, Z. Ficek, P. D. Drummond, N. R. Heckenberg, and H. Rubinsztein-Dunlop, "Light guiding light: Nonlinear refraction in rubidium vapor," Phys. Rev. A 63, 023820 (2001).

[12] D. H. Close, "Strong-field saturation effects in laser media," Phys. Rev. 153, 360-371 (1967).

[13] A. Yariv, Quantum Electronics, Wiley \& Sons, New York, (1989); P. N. Butcher and D. Cotter, The Elements of Nonlinear Optics, Cambridge University Press, Cambridge (1990). 
[14] M. Sheik-Bahae, A. A. Said and E. W. Van Stryland, "High-sensitivity, single-beam $n_{2}$ measurements," Opt. Lett. 14, 955-957 (1989); M. Sheik-Bahae, A. A. Said, T. H. Wei, D. J. Hagan and E. W. Van Stryland, “Sensitive measurement of optical nonlinearities using a single beam,” IEEE J. Quantum Electr. QE-26, 760-769 (1990).

[15] M. Abramowitz and I. A. Stegun, eds. Handbook of Mathematical Functions (U.S. Government Printing Office, Washington, D.C., 1968), p. 298.

[16] A. E. Siegman, “Quasi fast Hankel transform,” Opt. Lett. 1, 13-15 (1977); G. P. Agrawal and M. Lax, "End correction in the quasi-fast Hankel transform for optical-propagation problems," Opt. Lett. 6, 171-173 (1981). 